\title{
Prognostic factors for survival with nab- paclitaxel plus gemcitabine in metastatic pancreatic cancer in real-life practice: the ANICE-PaC study
}

Ana Fernández ${ }^{1 *}$, Mercedes Salgado ${ }^{2}$, Adelaida García $^{3}$, Elvira Buxò ${ }^{4}$, Ruth Vera ${ }^{5}$, Jorge Adeva ${ }^{6}$, Paula Jiménez-Fonseca ${ }^{7}$, Guillermo Quintero ${ }^{8}$, Cristina Llorca ${ }^{9}$, Mamen Cañabate ${ }^{10}$, Luis Jesús López ${ }^{11}$, Andrés Muñoz ${ }^{12}$, Patricia Ramírez ${ }^{13}$, Paula González ${ }^{14}$, Carlos López ${ }^{15}$, Margarita Reboredo ${ }^{16}$, Elena Gallardo ${ }^{17}$, Manuel Sanchez-Cánovas ${ }^{18}$, Javier Gallego ${ }^{19}$, Carmen Guillén ${ }^{20}$, Nuria Ruiz-Miravet ${ }^{21}$, Víctor Navarro-Pérez ${ }^{22}$, Juan De la Cámara ${ }^{23}$, Inmaculada Alés-Díaz ${ }^{24}$, Roberto Antonio Pazo-Cid ${ }^{25}$ and Alberto Carmona-Bayonas ${ }^{18}$

\begin{abstract}
Background: Treatment with nab-paclitaxel plus gemcitabine increases survival in patients with metastatic pancreatic cancer. However, the assessment of treatment efficacy and safety in non-selected patients in a real-life setting may provide useful information to support decision-making processes in routine practice.

Methods: Retrospective, multicenter study including patients with metastatic pancreatic cancer, who started firstline treatment with nab-paclitaxel plus gemcitabine between December 2013 and June 2015 according to routine clinical practice. In addition to describing the treatment pattern, overall survival (OS) and progression-free survival (PFS) were assessed for the total sample and the exploratory subgroups based on the treatment and patients' clinical characteristics.

Results: All 210 eligible patients had a median age of 65.0 years (range 37-81). Metastatic pancreatic adenocarcinoma was recurrent in 46 (21.9\%) patients and de novo in 164 (78.1\%); 38 (18\%) patients had a biliary stent. At baseline, 33 (18.1\%) patients had an ECOG performance status $\geq 2$. Patients received a median of four cycles of treatment (range 1-21), with a median duration of 3.5 months; 137 (65.2\%) patients had a dose reduction of nab-paclitaxel and/or gemcitabine during treatment, and 33 (17.2\%) discontinued treatment due to toxicity. Relative dose intensity (RDI) for nab-paclitaxel, gemcitabine, and the combined treatment was $66.7 \%$. Median OS was 7.2 months (95\% Cl 6.0-8.5), and median PFS was 5.0 months (95\% Cl 4.3-5.9); 50 patients achieved either a partial or complete response (ORR 24.6\%). OS was influenced by baseline ECOG PS, NLR and CA 19.9, but not by age $\geq 70$ years and/or the presence of hepatobiliary stent or $\mathrm{RDI}<85 \%$. All included variables, computed as dichotomous, showed a significant contribution to the Cox regression model to build a nomogram for predicting survival in these patients: baseline ECOG $0-1$ vs. $2-3(p=0.030)$, baseline NLR $>3$ vs. $\leq 3(p=0.043)$, and baseline CA $19.9>37 \mathrm{U} / \mathrm{mL}$ vs. $\leq 37 \mathrm{U} / \mathrm{mL}(p=0.004)$.

(Continued on next page)
\end{abstract}

\footnotetext{
* Correspondence: ana.fernandez.montes@sergas.es

${ }^{1}$ Complejo Hospitalario Universitario Ourense, Calle Ramon Puga Noguerol,

54, 32005 Ourense, Spain

Full list of author information is available at the end of the article
}

(c) The Author(s). 2018 Open Access This article is distributed under the terms of the Creative Commons Attribution 4.0 International License (http://creativecommons.org/licenses/by/4.0/), which permits unrestricted use, distribution, and reproduction in any medium, provided you give appropriate credit to the original author(s) and the source, provide a link to the Creative Commons license, and indicate if changes were made. The Creative Commons Public Domain Dedication waiver (http://creativecommons.org/publicdomain/zero/1.0/) applies to the data made available in this article, unless otherwise stated. 
(Continued from previous page)

Conclusions: Nab-Paclitaxel plus gemcitabine remain effective in a real-life setting, despite the high burden of dose reductions and poorer performance of these patients. A nomogram to predict survival using baseline ECOG performance status, NLR and CA 19.9 is proposed.

Keywords: Metastatic pancreatic adenocarcinoma, Gemcitabine, Nab-paclitaxel, Real-life, First-line chemotherapy, Survival

\section{Background}

Pancreatic adenocarcinoma, the most common form of pancreatic cancer, is currently the fourth cause of cancer-related mortality in Europe and the United States, with a 5-year survival rate in the range of $6-10 \%$ [1-4]. This has been attributed, among other causes, to the premature vascular, lymphatic and perineural spread of these tumors, which makes that $85 \%$ of patients present disseminated disease at diagnosis. Only between 15 and $20 \%$ of tumors are resectable and, within these, 50 to $86 \%$ will experience local failure despite curative resection, with a resulting 5-year survival rate of $10-20 \%$ [5].

Gemcitabine has been the standard first-line treatment for advanced pancreatic cancer for 15 years, and it is associated with median overall survivals (OS) ranging from 5.6 to 6.8 months [6]. Its combination with a wide range of other agents such as capecitabine, oxaliplatin, cisplatin, irinotecan, and erlotinib has unfortunately shown little impact on survival in this population $[6,7]$. Nonetheless, two combination regimens, FOLFIRINOX (folinic acid, fluorouracil, irinotecan, oxaliplatin) and more recently, nab-paclitaxel plus gemcitabine, have been associated with a median OS of 11.1 months and 8.5 months in the ACCORD4/ PRODIGE11 and MPACT phase III clinical trials, respectively $[8,9]$. These encouraging results have set a new standard and international guidelines now recommend FOLFIRINOX and nab-paclitaxel plus gemcitabine as first-line treatments in patients with metastatic pancreatic cancer $[10,11]$.

A questionnaire-based study showed that clinicians are likely to adhere to the selection criteria of these trials, being the patient's performance status one of the most influential factors in the decision-making process in the real-life setting [12]. As randomized trials have strict selection criteria, particularly regarding performance status and age $[8,9]$, reported data might not capture the scenario faced in the real-life setting, with non-selected patients. A prospective registry-based study showed that fewer than half of the patients treated in routine clinical practice would have been eligible for the PRODIGE or MPACT trials and that meeting the eligibility criteria for either trial is associated with longer survival [13].

Clinicians are increasingly interested in obtaining real-life data from daily practice, which completes the clinical picture of randomized trials. As such, observational trials can provide useful information for clinicians to support decision-making processes in their routine practice, especially when treating patient groups underrepresented in clinical trials, as are elderly patients and those with poorer performance status $[14,15]$. In line with this unmet need, Ellenrieder et al. highlighted the importance of real-life data regarding first-line regimens in metastatic pancreatic cancer to select the most suitable treatment for each patient [7]. The aim of the ANICE study was to assess the effectiveness and tolerability of gemcitabine and nab-paclitaxel as first-line therapy for metastatic pancreatic cancer in a real-life setting.

\section{Methods}

\section{Study design and patients}

The ANICE trial was an observational, retrospective, multicenter study focused on patients with metastatic pancreatic adenocarcinoma (recurrent or de novo) treated according to routine clinical practice at 20 Spanish hospitals between December 2013 and June 2015. All adult patients ( $\geq 18$ years) with measurable metastatic disease at baseline in at least one dimension (per Response Evaluation Criteria in Solid Tumors [RECIST], version 1.1, [16]) who received at least one dose of nab-paclitaxel (Abraxane ${ }^{\bullet}$, Celgene Europe Limited) plus gemcitabine as first-line chemotherapy were included. Data were obtained from clinical medical records with a cut-off date of 16 March 2017. All patients provided written informed consent. The study protocol was approved by the local independent ethics committee, and was conducted in accordance with the Spanish personal data protection law (LOPD 15/1999).

\section{Variables and endpoints}

Variables collected from the patient's medical records included demographic data (sex and age), clinical data, characteristics of the metastatic disease and treatment. Among the registered clinical and disease characteristics were performance status (PS) (ECOG and Karnofsky scales), relevant comorbidities, initial diagnosis, number and localization of metastases, time between diagnosis of the primary tumor and recurrence, presence of a hepatobiliary stent, serum bilirubin, neutrophil/lymphocyte 
ratio (NLR), and CA 19.9 antigen levels. Treatment characteristics included concomitant treatments, relative dose intensity (RDI) for nab-paclitaxel plus gemcitabine (i.e., per the summary of product characteristics), treatment duration, cause of treatment discontinuation, number of cycles, and dose reductions and interruptions. Decisions regarding the initial dose and subsequent dose reductions were made at physicians' discretion, based on patient's PS and toxicity, and following the routine practice of each participating sit.

The primary objective was to describe the treatment pattern in terms of extent of exposure and reductions of treatment with nab-paclitaxel plus gemcitabine in real-life clinical practice. Secondary objectives included objective response rate (ORR), progression-free survival (PFS) defined as the time from the start of treatment to disease progression or all-cause death, overall survival (OS) defined as the time from the start of treatment to death from any cause, and the 12-month survival rate defined as the percentage of patients alive at 12 months after starting treatment. Safety was assessed in terms of adverse events $(\mathrm{AE})$, coded according to the preferred term of the Medical Dictionary for Regulatory Activities (MedDRA), and graded according to the National Cancer Institute-Common Toxicity Criteria (version 4.0) [17].

\section{Statistical analyses}

Given the descriptive nature of the statistical analyses, the sample size was calculated based on the confidence interval $(\mathrm{CI})$ of the 12-month survival rate. Considering the 12-month survival rate previously reported by Goldstein et al. [18], and assuming that nearly $20 \%$ of patients present with recurrent metastatic cancer, a sample of 225 patients was deemed necessary to estimate a 12 -month survival rate of $30 \%$ with a $\pm 6 \%$ precision and a $95 \% \mathrm{CI}$.

Categorical variables were summarized as frequencies and percentages, and quantitative variables as the mean and standard deviation (SD) and/or median and interquartile range (IQR). The quantitative variables NLR and CA 19.9 were transformed into dichotomous variables with the cut-offs values of 3 and $37 \mathrm{U} / \mathrm{mL}$, respectively. Categorical values were compared using the Fisher's exact test or the chi-square test when the requirements for a Fisher's exact test could not be assumed. Quantitative values were compared using the T-test, ANOVA test, and their non-parametric counterparts, the Wilcoxon and Kruskal-Wallis tests. OS and PFS curves were plotted using the Kaplan-Meier estimate, and compared according to selected parameters using the Log-rank test and the Cox regression model. The significance threshold for all bivariate analyses was set at a two-sided $\alpha=0.05$. All factors showing a significant influence on OS in the bivariate analysis were included in a Cox regression model as dichotomous variables to build a nomogram for predicting OS in real-life practice. The obtained hazard ratios (HR) were used to associate each variable with a survival score. In addition to the predicted survival, a probability for 3-, 6-, 12, and 18-month survival was estimated. Based on the range of final scores resulting from the possible combinations of variables, low-, medium-, and high-risk groups were defined. All analyses were performed using the statistical package SAS system for Windows version 9.4.

\section{Results}

\section{Patient characteristics}

Of the 216 patients recruited, 6 were excluded for one or more of the following reasons: not initiating combined treatment with gemcitabine plus nab-paclitaxel $(n=4)$, absence of measurable disease at baseline per RECIST $(n=2)$, and being enrolled in a clinical trial $(n=1)$. Table 1 summarizes the demographic and clinical characteristics of study patients. The 210 eligible patients had a median age of 65.0 years (range 37-81). Metastatic pancreatic adenocarcinoma was recurrent in $46(21.9 \%)$ patients and de novo in 164 (78.1\%). For patients with recurrent metastatic disease, the primary tumor was resectable in 38 cases $(82.6 \%)$, borderline in 3 (6.5\%), and locally advanced unresectable in 5 (10.9\%), and median time to recurrence was 11.0 months. Assessment of eligibility for the MPACT and ACCORD4/ PRODIGE11 trials could be assessed in 78 (37.1\%) and $172(81.9 \%)$ patients, respectively, with 37 meeting the criteria for entering the MPACT trial and 109 the ACCORD4/PRODIGE11 trial.

\section{Treatment characteristics and outcome}

Table 2 summarizes treatment characteristics. Median time from diagnosis to treatment start was 28 days. Patients received a median of 4 cycles (range 1-21) of treatment, with a median treatment duration of 3.5 months. Sixty-eight patients (32\%) started treatment with dose reduction for either nab-paclitaxel, gemcitabine or both drugs. Table 3 summarizes the baseline characteristics of the 68 patients with dose reduction and treatment start. The median RDIs were $66.7 \%$ for nab-paclitaxel, gemcitabine, and the combined treatment. Overall, 137 patients (65.2\%) had a dose reduction in nab-paclitaxel and/or gemcitabine during treatment. Thirty-four (17\%) patients received $\leq 30$ days of treatment, mainly due to toxicity $(n=11)$ or disease progression $(n=10)$. There were no significant differences between patients receiving $\leq 30$ days of treatment and those receiving $>30$ days in terms of baseline clinical characteristics including performance status, the presence of a hepatobiliary stent, 
Table 1 Demographic and clinical characteristics of study patients

\begin{tabular}{|c|c|c|c|c|}
\hline & Number $^{a}$ & Overall & Recurrent & De novo \\
\hline \multicolumn{5}{|l|}{ Demographic characteristics } \\
\hline Age, $n(\%)$ & 210 & & & \\
\hline$<65$ years & & 99 (47.1\%) & $22(47.8 \%)$ & 77 (47.0\%) \\
\hline $65-69$ years & & $58(27.6 \%)$ & $8(17.4 \%)$ & $50(30.5 \%)$ \\
\hline$\geq 70$ years & & $53(25.2 \%)$ & $16(34.8 \%)$ & $37(22.6 \%)$ \\
\hline Sex, $n(\%)$ & 210 & & & \\
\hline Males & & $127(60.5)$ & $33(71.7)$ & $94(57.3)$ \\
\hline Females & & $83(39.5)$ & $13(28.3)$ & $70(42.7)$ \\
\hline \multicolumn{5}{|l|}{ Clinical characteristics } \\
\hline Weight loss > 10\%, n (\%) & 208 & $81(38.9)$ & $9(20.5)$ & $72(43.9)$ \\
\hline ECOG PS, $n(\%)$ & 182 & & & \\
\hline $0-1$ & & $149(81.9)$ & $28(77.8)$ & $121(82.9)$ \\
\hline $2-3$ & & $33(18.1)$ & $8(22.2)$ & $25(17.1)$ \\
\hline Karnofsky PS, n (\%) & 55 & & & \\
\hline$<70$ & & $3(5.5)$ & - & $3(6.8)$ \\
\hline $70-80$ & & $33(60.0)$ & $7(63.6 \%)$ & $26(59.1 \%)$ \\
\hline $90-100$ & & $19(34.5)$ & $4(36.4 \%)$ & $15(34.1 \%)$ \\
\hline Common comorbidities, $n$ (\%) & 210 & & & \\
\hline Hypertension & & $56(26.7)$ & $8(17.4)$ & $48(29.3)$ \\
\hline Diabetes & & $26(12.4)$ & $4(8.7)$ & $22(13.4)$ \\
\hline Dyslipemia & & $29(13.8)$ & $6(13.0)$ & $22(13.4)$ \\
\hline Hepatobiliary stent, $n$ (\%) & 209 & $38(18.2)$ & $3(6.5)$ & $35(21.5)$ \\
\hline Platelet count, median (IR) & 178 & $231.0(172.0,318.0)$ & $207.0(170.0,303.0)$ & $237.0(181.0,318.0)$ \\
\hline Bilirubin (mg/dL), median (IR) & 175 & $0.70(0.50,1.00)$ & $0.57(0.50,0.80)$ & $0.79(0.50,1.10)$ \\
\hline NLR, $n(\%)$ & 170 & & & \\
\hline$>3$ & & $91(53.5)$ & $18(52.9)$ & $73(53.7)$ \\
\hline$\leq 3$ & & $79(46.5)$ & $16(47.1)$ & $63(46.3)$ \\
\hline CA $19.9, n(\%)$ & 173 & & & \\
\hline$>37 \mathrm{U} / \mathrm{mL}$ & & $142(82.1)$ & $32(82.1)$ & $110(82.1)$ \\
\hline$\leq 37 \mathrm{U} / \mathrm{mL}$ & & $31(17.9)$ & $7(17.9)$ & $24(17.9)$ \\
\hline Number of metastatic sites, $n$ (\%) & 210 & & & \\
\hline $1-3$ & & $205(97.6)$ & $46(100.0)$ & $159(97.0)$ \\
\hline$>3$ & & $5(2.4)$ & & $5(3.0)$ \\
\hline Concommitant treatment, $n$ (\%) & 210 & & & \\
\hline Analgesics & & $76(36.2)$ & $12(26.1)$ & $63(38.4)$ \\
\hline Corticosteroids & & $25(11.9)$ & $4(8.7)$ & $20(12.2)$ \\
\hline
\end{tabular}

$I R$ Interquartile range (percentile 25, percentile 75 )

${ }^{a}$ number of evaluable patients (no-missing)

NLR, CA 19.9 and bilirubin levels, or weight loss of more than $10 \%$.

At the time of data collection, median follow-up was 7.2 months (IQR 3.5-13.3). A total of 193 patients (91.9\%) had died, with a 12-month survival rate of $30.1 \%$. Median OS was 7.2 months (95\% CI 6.0-8.5), and median PFS 5.0 months (95\%CI 4.3-
5.9). Among 203 patients eligible for response, 50 patients achieved either a partial or complete response (ORR 24.6\%), and 72 patients (35.5\%) a stable disease. Ninety-seven patients $(46.9 \%)$ received further treatment lines, most of whom $(n=63$, $64.9 \%)$ had one or two lines of treatment $(n=25$, $25.8 \%)$. 
Table 2 Treatment characteristics

\begin{tabular}{ll}
\hline & No. (\%) \\
\hline Started treatment with dose reduction, $n$ (\%) & $1(0.5)$ \\
Only gemcitabine & $41(19.5)$ \\
Only nab-Paclitaxel & $26(12.4)$ \\
Both & \\
Dose reduction during treatment, $n$ (\%) & $91(43.3)$ \\
Nab-Paclitaxel & $75(35.7)$ \\
Gemcitabine & $96(45.7)$ \\
Either of the two drugs & $34(16.5)$ \\
Received $\leq 30$ days of treatment, $n(\%)$ & \\
Reasons for treatment discontinuation ${ }^{\mathrm{a}}, n$ (\%) & $134(69.8)$ \\
Progression & $33(17.2)$ \\
Toxicity & $24(12.5)$ \\
Death & $7(3.6)$ \\
Patient's request
\end{tabular}

${ }^{\mathrm{a}} \mathrm{A}$ patient could have more than one reason for treatment discontinuation

\section{Safety}

Grade $\geq 3$ treatment-related AEs were reported in 78 patients (37.1\%), the most common being neutropenia, thrombocytopenia, and fatigue (Table 4). One treatment-related event of paralytic ileus led to treatment interruption and death.

Patients aged $<70$ years received a median of 5 cycles of combined treatment and patients aged $\geq 70$ years received a median of 3 . Of 53 elderly patients (i.e., aged $\geq 70$ years), 38 (71.7\%) experienced at least one treatment-related $\mathrm{AE}$, compared to $78.1 \%$ in the overall population, with similar profile of grade $\geq 3$ events (Table 4). No significant differences were found in the frequency of treatment-related AEs of grade 3 or higher between patients aged $\geq 70$ years and $<70(39.6 \%$ vs. $36.3 \% ; p=0.789)$.

\section{Prognostic factors}

Analysis of the influence of baseline characteristics on survival showed that only ECOG, NLR, and CA 19.9 significantly influenced OS, PFS (Fig. 1), and/or 12-month survival. Baseline CA 19.9 had a significant influence on the three analyzed outcomes: patients with CA $19.9 \leq 37$ $\mathrm{U} / \mathrm{mL}$ had longer OS $(p=0.004)$ (Fig. 1e), PFS $(p=$ 0.011 ) (Fig. 1f), and a higher 12-month survival rate $(45.2 \%$ vs. $24.8 \% ; p=0.030)$. On the other hand, patients with baseline NLR $\leq 3$ had longer OS $(p=0.024)$ (Fig. 1c) and a higher 12-month survival rate than those with NLR > 3 (38\% vs. $23.3 \%$; $p=0.045)$ but showed similar PFS (Fig. 1d). Patients with a baseline ECOG PS of 0 or 1 had longer OS than those with an ECOG PS of 2 or 3 $(p=0.018)$ (Fig. 1a), although this trend was not observed in PFS (Fig. 1b). Likewise, the 12-month survival for patients with ECOG PS 0 or 1 and 2 or 3 was 31.1
Table 3 Demographic and clinical characteristics of patients who started treatment with dose reduction

\begin{tabular}{|c|c|c|}
\hline \multicolumn{3}{|l|}{ Demographic characteristics } \\
\hline Age, $n(\%)$ & $(n=68)$ & \\
\hline$<65$ years & & $33(48.5 \%)$ \\
\hline $65-79$ years & & $14(20.6 \%)$ \\
\hline$\geq 70$ years & & $21(30.9 \%)$ \\
\hline Sex, $n(\%)$ & $(n=68)$ & \\
\hline Males & & $41(60.3 \%)$ \\
\hline Females & & 27 (39.7\%) \\
\hline \multicolumn{3}{|l|}{ Clinical characteristics } \\
\hline Weight loss > 10\%, n (\%) & $(n=66)$ & $18(27.3 \%)$ \\
\hline ECOG PS, $n(\%)$ & $(n=62)$ & \\
\hline $0-1$ & & $46(74.2 \%)$ \\
\hline 2-Mar & & $16(25.8 \%)$ \\
\hline Karnofsky PS, n (\%) & $(n=23)$ & \\
\hline$<70$ & & - \\
\hline $70-80$ & & $13(56.5 \%)$ \\
\hline $90-100$ & & $10(43.5 \%)$ \\
\hline Common comorbidities, $n$ (\%) & $(n=68)$ & \\
\hline Hypertension & & $15(22.1 \%)$ \\
\hline Diabetes & & $8(11.8 \%)$ \\
\hline Dyslipemia & & $4(5.9 \%)$ \\
\hline Hepatobiliary stent, $n$ (\%) & $(n=68)$ & $10(14.7 \%)$ \\
\hline Platelet count, median (IR) & $(n=57)$ & $252.0(172.0,339.0)$ \\
\hline Bilirubin (mg/dL), median (IR) & $(n=58)$ & $0.70(0.51,1.10)$ \\
\hline$N L R, n(\%)$ & $(n=55)$ & \\
\hline$>3$ & & $29(52.7 \%)$ \\
\hline$\leq 3$ & & $26(47.3 \%)$ \\
\hline CA $19.9, n(\%)$ & $(n=62)$ & \\
\hline$>35$ & & $52(83.9 \%)$ \\
\hline$\leq 35$ & & $10(16.1 \%)$ \\
\hline Number of metastatic sites, $n(\%)$ & $(n=68)$ & \\
\hline $1-3$ & & 67 (98.5\%) \\
\hline$>3$ & & $1(1.5 \%)$ \\
\hline Concommitant treatment, $n$ (\%) & $(n=68)$ & \\
\hline Analgesics & & 22 (32.4\%) \\
\hline Corticosteroids & & $9(13.2 \%)$ \\
\hline
\end{tabular}

IR Interquartile range (percentile 25, percentile 75)

a number of evaluable patients (no-missing)

and $21.2 \%$, respectively $(p=0.297)$. Patients with stable ECOG $(n=68)$ or ECOG improvement $(n=22)$ during the first three treatment cycles had longer OS than those with a worsening ECOG $(n=19)$, with a median OS of 12.9 months (95\% CI 6.7-15.4), 10.6 months (95\% CI 8.1-13.3), and 7.1 months (95\% CI 4.7-9.2) 
Table 4 Common treatment-related adverse events (> 1\% of patients overall) of grade 3. No. (\%)

\begin{tabular}{llll}
\hline & $\begin{array}{l}\text { Overall } \\
(n=210)\end{array}$ & $\begin{array}{l}<70 \text { years } \\
(n=157)\end{array}$ & $\begin{array}{l}\geq 70 \text { years } \\
(n=53)\end{array}$ \\
\hline Hematological toxicities & & & \\
Neutropenia & $38(18.1)$ & $33(21.0)$ & $5(9.4)$ \\
Thrombocytopenia & $13(6.2)$ & $9(5.7)$ & $4(7.5)$ \\
Anemia & $7(3.3)$ & $2(1.3)$ & $5(9.4)$ \\
Febrile neutropenia & $4(1.9)$ & $2(1.3)$ & $2(3.8)$ \\
Non-hematological toxicities & & & \\
Fatigue & $13(6.2)$ & $10(6.4)$ & $3(5.7)$ \\
Vomiting & $3(1.4)$ & $1(0.6)$ & $2(3.8)$ \\
Colangitis & $3(1.4)$ & $3(1.9)$ & - \\
Neurotoxicity & $3(1.4)$ & $1(0.6)$ & $2(3.8)$ \\
Peripheral neuropathy & $5(2.4)$ & $3(1.9)$ & $2(3.8)$ \\
Alopecia & $9(4.3)$ & $6(3.8)$ & $3(5.7)$ \\
\hline
\end{tabular}

for ECOG improvement, stable, and worsening, respectively $(p=0.020)$.

Neither age $\geq 70$ years, the presence of an hepatobiliary stent, nor an RDI of gemcitabine plus nab-paclitaxel $<85 \%$ showed a significant influence on median PFS and OS at 12 months (Fig. 2). The 12-month survival rate was 28.3 and $30.8 \%$ for patients aged $\geq 70$ and $<70$ years, respectively ( $p=0.863$ ); 36.8 and $28.8 \%$ for patients with and without hepatobiliary stent, respectively $(p=0.335)$; and 28.0 and $31.5 \%$ for patients with RDI of the combined treatment $\geq 85$ and $<85 \%$, respectively ( $p=0.645$ ).

Factors significantly influencing OS (ECOG PS, NLR, and CA 19.9) were used to build a nomogram to predict survival of patients with pancreatic adenocarcinoma treated in the real-life setting (Fig. 3a). All included variables, computed as dichotomous, showed a significant contribution to the Cox regression model: baseline ECOG PS 0 or 1 vs 2 or $3(p=0.030)$, baseline NLR $>3$ vs $\leq 3(p=0.043)$, and baseline CA $19.9>37 \mathrm{U} / \mathrm{mL}$ vs $\leq 37 \mathrm{U} / \mathrm{mL}(p=0.004)$ (Fig. 3a). Based on the scale obtained with the nomogram, three risk groups were defined: low-risk group $(n=21,15.1 \%)$, medium-risk group $(n=93,66.9 \%)$, and high-risk group $(n=25,18.0 \%)$. Figure $3 \mathrm{~b}$ shows the survival curves (Kaplan-Meier estimates for these groups).

\section{Discussion}

In this observational retrospective study including all the patients with metastatic pancreatic cancer treated at the participating centers with first-line nab-paclitaxel plus gemcitabine in a real-life setting, $25 \%$ of patients were aged 70 years or more and $18 \%$ had a baseline ECOG score of 2 or 3 . Furthermore, 32\% of patients started combined treatment with a dose reduction of nab-paclitaxel, and $17 \%$ of patients discontinued treatment within 30 days of treatment, $33 \%$ of them due toxicity. Despite the poor baseline characteristics in our cohort, median OS and PFS were of 7.2 months (95\% CI $6.0,8.5)$ and 5.0 months $(4.3,5.9)$, respectively, indicating that this combination is effective in the real-life setting. OS was influenced by the baseline ECOG PS, NLR, and CA 19.9, but not by age $\geq 70$ years, the presence of hepatobiliary stent or RDI $<85 \%$.

Randomized controlled trials investigating therapies for metastatic pancreatic cancer have restrictive selection criteria, particularly regarding the patient's age and performance status $[8,9]$. In the case of the pivotal study of the combined treatment (MPACT trial), a Karnofsky index of 70 or more was required [9]. In Spain the preferred scale to assess the performance status in most centers is the ECOG score, thus, in our study, the Karnofsky index could only be assessed in a limited number of patients. However, although the Karnofsky index and the ECOG score lack a linear relationship for direct comparisons between study populations, the fact that $18 \%$ of patients had ECOG $\geq 2$ (roughly $\geq 70$ in the Karnofsky index) indicates a trend towards a poorer average performance status in our study population than those in the pivotal MPACT trial. In the case of the ACCORD4/PRODIGE11 study, recruitment was limited to patients under 76 years with ECOG performance status $\leq 1$; [8] based on these criteria alone, nearly $40 \%$ of our study patients would have been excluded. In addition to the patients' baseline characteristics, treatment patterns in real-life practice often differ from those used in pivotal trials. In our study, $66.2 \%$ of patients had a dose reduction in either nab-paclitaxel and/or gemcitabine. Overall, the median RDI was $67 \%$ for both nab-paclitaxel and gemcitabine, which is well below those reported in the MPACT trial (81 and $75 \%$ for nab-paclitaxel and gemcitabine, respectively). Thus, the demographic, clinical and treatment characteristics of this large cohort of real-life patients underscore fundamental differences between RCTs and routine practice settings.

Despite the inclusion of elderly patients, the trend towards a poorer performance status, and adjusted treatment schedule in our study, our results confirmed the effectiveness of combined treatment with gemcitabine plus nab-paclitaxel in the real-life setting. The estimated median OS (7.2 months) was slightly lower than that observed in the MPACT trial (8.5 months) and those reported by Giordano et al. (11 months), Lo Re et al. (9.2 months), and De Vita et al. (10 months) in three series of 118,37 , and 41 real-life patients, respectively [19-21]. Likewise, the median PFS of our cohort (5.0 months) was comparable to that reported in the MPACT trial (5.5 months), but lower than that reported by Giordano et al. (7 months), Lo $\operatorname{Re}$ et al. (6.2 months), and of 


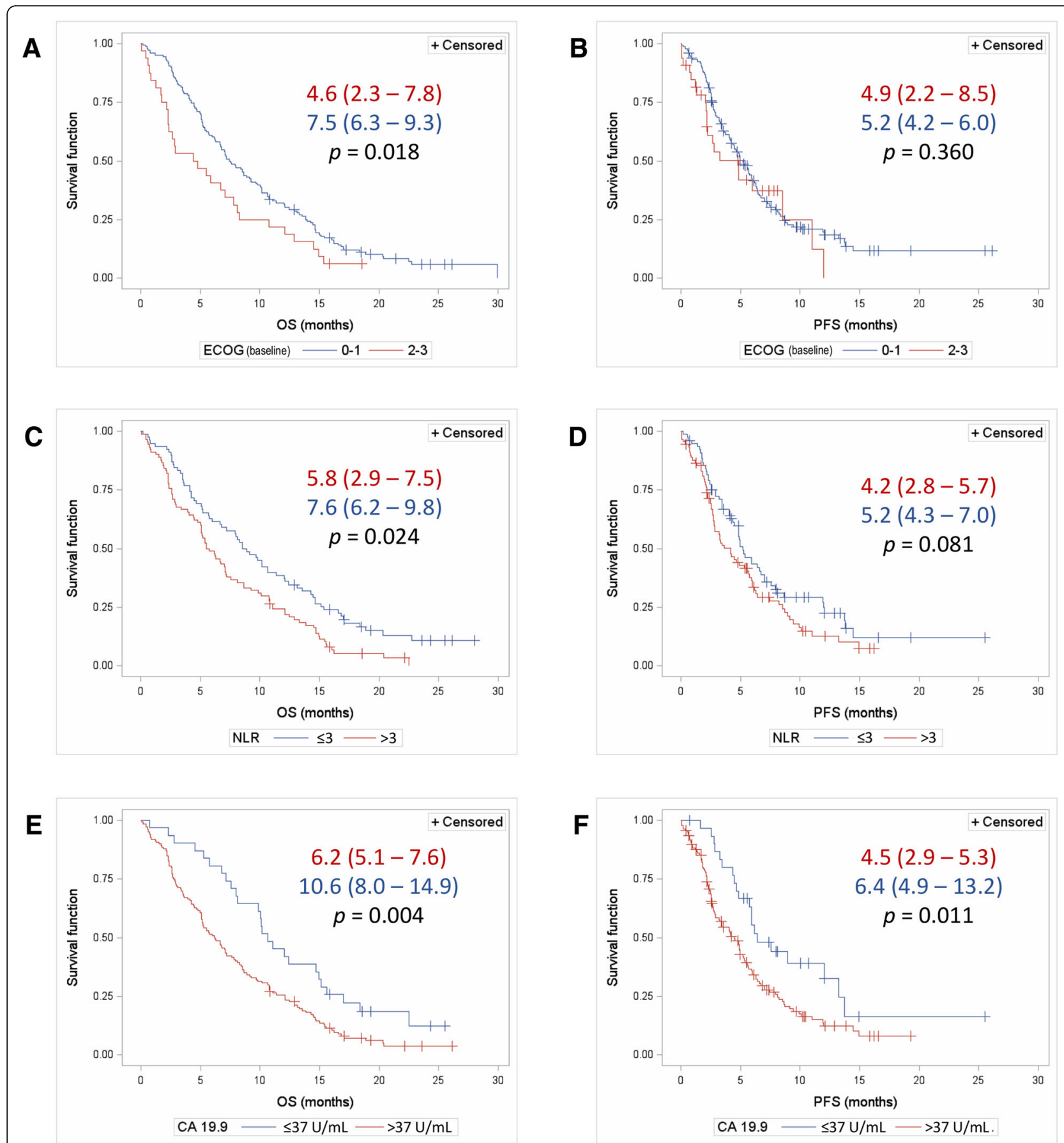

Fig. 1 Overall survival (a, $\mathbf{c}$ and $\mathbf{e}$ ) and progression-free survival (b, $\mathbf{d}$ and $\mathbf{f}$ ) depending on baseline ECOG (a and b), NLR (c and $\mathbf{d})$ and CA 19.9 (e and $\mathbf{f}$ ). Survival is presented as median ( $95 \% \mathrm{Cl}$ ); $p$-values correspond to the Log-rank test for inter-curve differences

notably Da Vitta et al. (9.2 months). Of note, patients of our cohort had poorer PS than that reported in these studies.

As reported in previous studies, neutropenia was the most common treatment-related adverse event of grade 3 or higher, however its $18 \%$ incidence was substantially below that reported in the MPACT trial
(38\%) [9] and slightly lower than in previous series of real-life patients (20 to 24\%) [14, 19, 20]. Remarkably, being aged over 70 years was not associated with a worse toxicity profile in our study, consistent with the trend reported by Giordano et al. in a retrospective study addressing the safety of this treatment in the elderly [14]. 


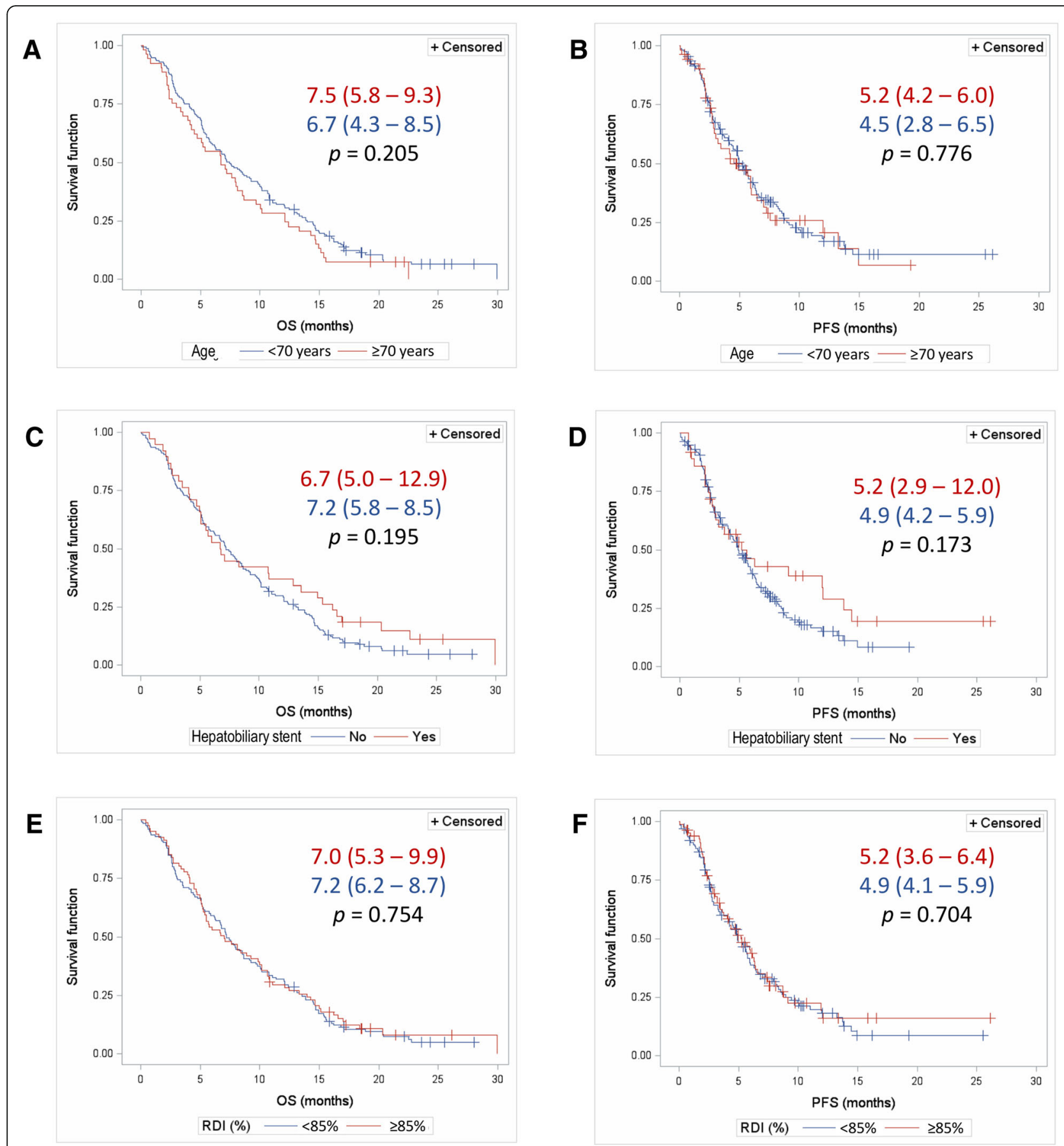

Fig. 2 Overall survival (a, c and e) and progression-free survival (b, $\mathbf{d}$ and $\mathbf{f}$ ) depending on baseline age (a and $\mathbf{b}$ ), presence of hepatobiliary stent (c and $\mathbf{d}$ ), and relative dose intensity (RDI) (e and $\mathbf{f}$ ). Survival is presented as median ( $95 \% \mathrm{Cl}$ ); p-values correspond to the Log-rank test for inter-curve differences

In addition to assessing the safety and effectiveness of first-line nab-paclitaxel plus gemcitabine in real-life patients, we also analyzed prognostic factors and their influence on survival in this setting. The influence of the patient's performance status has been consistently reported by various authors. [18, 19, 21, 22] As in previous analyses of real-life patients, $[19,21]$ stratifying patients according to the baseline ECOG score of 0-1 or $>1$, shows an influence of ECOG on OS. Similarly, the inflammation-based NLR score, identified as a prognostic factor for OS and PFS in patients receiving nab-paclitaxel plus gemcitabine in pivotal studies [18] 


\section{A}

Points

ECOG

CA19.9

NLR

Total Points

3-Month Survival Probability

6-Month Survival Probability

12-Month Survival Probability

18-month Survival Probability

Predicted Median Surival (months)

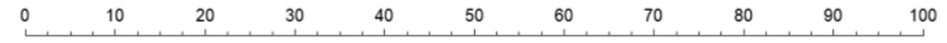

$2-3$

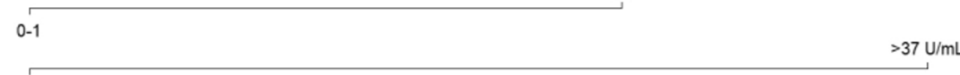

$<=37 \mathrm{U} / \mathrm{mL}$

$<=3$
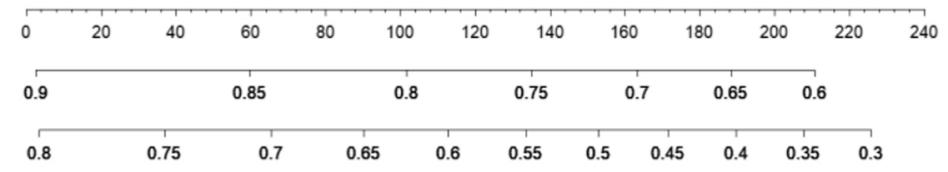

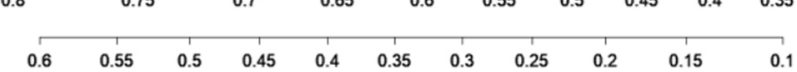

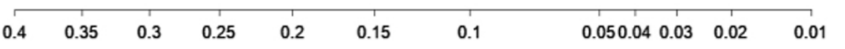

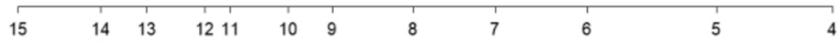

B

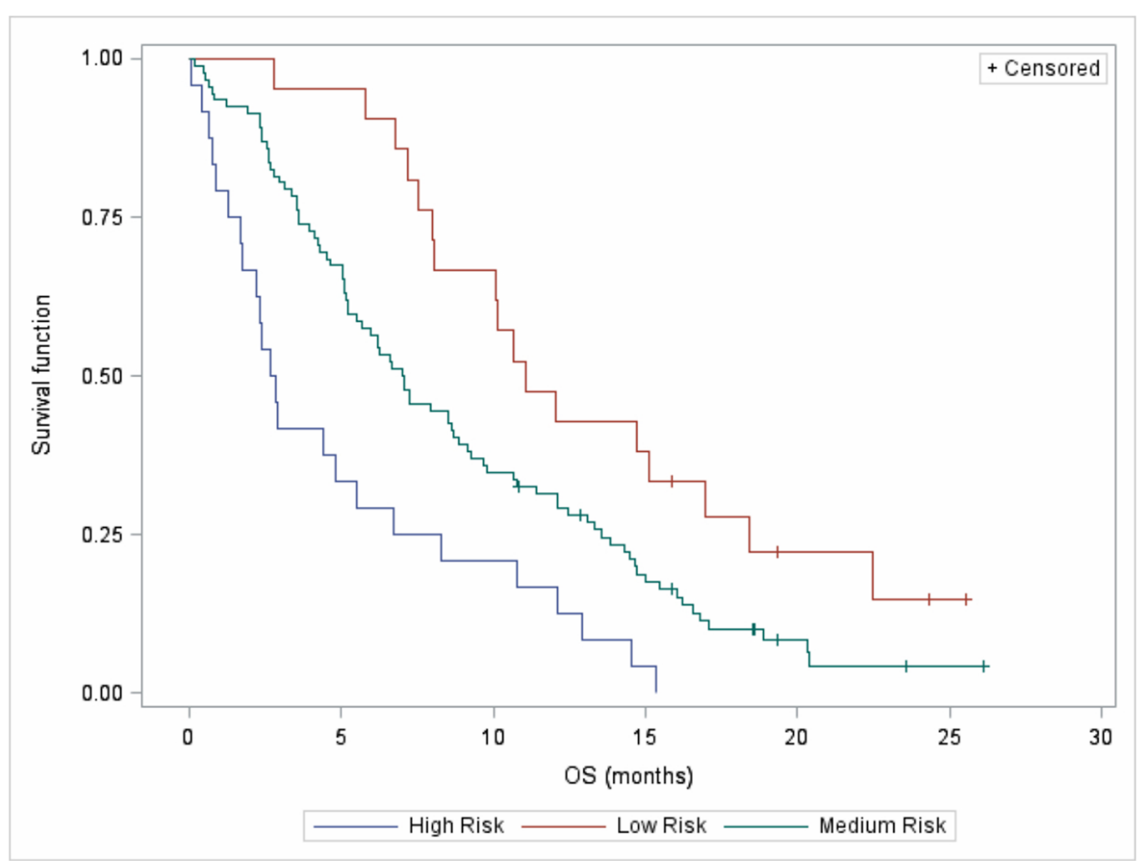

Fig. 3 Survival estimate of patients with metastatic pancreatic cancer starting combined treatment with nab-paclitaxel plus gemcitabine in real-life practice. a Nomogram for predicting overall survival and the probability of 3-month, 6-month, 12-month in real-life practice. $\mathbf{b}$ Survival (Kaplan Meier estimate) for low-, medium-, and high-risk groups

and real-life practice $[20,21,23]$, influenced OS in our patients but did not reach the significance threshold in the PFS analysis. In both localized and metastatic pancreatic adenocarcinoma, a proinflammatory status of the tumor results in worse prognosis, and therefore, influences treatment response and consequently, survival. Finally, the antigen CA 19.9 significantly influenced both
OS and PFS with a cut-off of $37 \mathrm{U} / \mathrm{mL}$. Other factors, such as age, the presence of a hepatobiliary stent, and the RDI did not significantly influence either OS or PFS. This finding is particularly controversial for age, which has been identified as a major prognostic factor for all patients with metastatic pancreatic cancer [22], and was subsequently confirmed for patients treated specifically 
with gemcitabine plus nab-paclitaxel [18]. It is worth noting that the cut-off age considered as a prognostic factor is not homogeneous across studies, and while risk analyses were traditionally based on patients over 60 or 65 years [18, 22], there is increasing interest in investigating patients over 70 years as a risk group in real-life practice [14, 24].

The prognostic factors identified in our cohort (i.e., ECOG PF, NLR, and CA 19.9) allowed us to develop a nomogram for predicting survival of real-life patients treated with first-line nab-paclitaxel plus gemcitabine. A similar tool for predicting survival in real-life patients receiving gemcitabine-based chemotherapy was presented by Hamada et al., and included age, sex, PS, tumor size, and the presence of nodal or distant metastases [25]. As in their analysis, PS carried a notable weight in our nomogram, with patients having an ECOG score of 2 or more dramatically reducing the predicted survival. On the other hand, variables not routinely assessed in standard practice, such as tumor size were not considered. In addition to the nomogram by Hamada et al., Goldstein et al. presented a similar tool based on the cohort of the MPACT trial [26]. The nomogram by Goldstein et al. was similar to ours in terms of the inclusion of performance status and NLR, but included other variables such as albumin, tumor size, and the presence of liver metastasis. Future studies shall validate the proposed nomogram as a tool for predicting survival in real-life patients.

Our results should be interpreted in the context of the intrinsic limitations of retrospective studies. Thus, in addition to the risk of reporting bias associated with observational designs, missing data in the medical records could not be considered for the analysis, leading uneven sample sizes across analyses. The retrospective design also precluded the inclusion of variables not recorded in routine clinical practice in Spain, notably the Karnofsky index, which was reported in very few patients and prevented a direct comparison with the study sample of the pivotal trial MPACT. Another limitation of the retrospective design was the lack of pre-defined criteria for dose reductions, which were established at physician's discretion, according to the routine practice in each center. Finally, the reduced size of some patient subgroups in the comparative analyses limited the investigation of baseline factors with potential influence on patient survival.

\section{Conclusions}

Our results, obtained from the largest published series of real-world patients with metastatic pancreatic cancer, show that nab-paclitaxel plus gemcitabine remains effective in this setting, despite the high burden of dose reductions and the poorer performance of these patients. Based on the exploratory analysis of prognostic factors, a nomogram was developed to predict survival of patients starting treatment with the combination. It was based on three variables routinely assessed in real-life practice, ECOG performance status, NLR, and CA 19.9.

\section{Abbreviations}

AE: Adverse events; Cl: Confidence interval; HR: Hazard ratio; IQR: Interquartile range; NLR: Neutrophil lymphocyte rate; ORR: Objective response rate; OS: Overall survival; PFS: Progression-free survival; PS: Performance status; RDI: Relative dose intensity; RECIST: Response Evaluation Criteria in Solid Tumors

\section{Acknowledgements}

The authors would like to thank the Galician Group for Research on Gastrointestinal Tumors (GITUD), as well as all study patients and their relatives. Statistical and medical writing support were provided by Josep Puig and Gerard Carot-Sans (PhD) on behalf of BioClever S.L.

\section{Funding}

The study coordination, data analysis, and medical writing assistance were supported by the non-profit Galician Group for Research on Gastrointestinal Tumors (GITUD) with funding from Celgene Corporation. The funding body was not directly involved in the study design, data analysis, and manuscript writing, which were performed by either the authors or the contract research organization BioClever, S.L. as stated in the acknowledgements section.

\section{Availability of data and materials \\ The datasets used and/or analyzed during the current study are available from the corresponding author on reasonable request.}

\section{Authors' contributions}

$A F$ and $M S$ significantly contributed to the study design. AF, AG, EB, RV, JA PJ, GQ, CLI, MC, LL, AM, PR, PG, CLó, MR, EG, MSC, AC, JG, CG, NR, VN, JC, IA, $R A P$, and $A C$ contributed to data collection, and $A F, A G, P J$, and $A C$ analyzed and interpreted the results. The manuscript was drafted by $A F, A G, J A, P J$, and $A C . A F, M S, A G, E B, R V, J A, P J, G Q, C L I, M C, L L, A M, P R, P G, C L o ́, M R, E G$, MSC, JG, CG, NR, VN, JC, IA, RAP, and AC revised the manuscript drafts critically and approved the final version of the manuscript.

\section{Ethics approval and consent to participate}

The study was approved by the ethics committee of the autonomous community of Galicia (SERGAS) (Spain). All patients provided written informed consent before data collection.

Consent for publication

Not applicable.

\section{Competing interests}

AG has received a grant for attending an international oncology congress and fees as scientific consultant from Celgene. AM has received research support grants and fees as consultant from Celgene, and fees as speaker from Shire. CL2 has received fees as speaker and consultant from Celgene. $A F, M S, E B, R V, J A, P J, G Q, C L 1, L L, P R, P G, M R, E G, M S C, A C, J G, C G, N R$, VN, $J C, I A, R A P$ declare that they have no conflicts of interest regarding the content of this manuscript.

\section{Publisher's Note}

Springer Nature remains neutral with regard to jurisdictional claims in published maps and institutional affiliations.

\section{Author details}

${ }^{1}$ Complejo Hospitalario Universitario Ourense, Calle Ramon Puga Noguerol, 54, 32005 Ourense, Spain. ${ }^{2}$ Complejo Universitario Ourense, Ourense, Spain. ${ }^{3}$ Institut Català d'Oncologia (ICO) Hospital Dr. Trueta, Girona, Spain. ${ }^{4}$ Hospital Clínic de Barcelona, Barcelona, Spain. ${ }^{5}$ Complejo Hospitalario de Navarra, Navarra, Spain. ${ }^{6}$ Hospital 12 de Octubre, Madrid, Spain. ${ }^{7}$ Hospital Universitario Central de Asturias, Asturias, Spain. ${ }^{8}$ Hospital Lucus Agustí, Lugo, Spain.

${ }^{9}$ Hospital de Elda, Alicante, Spain. ${ }^{10}$ Hospital Público Lluis Alcanyis de Xátiva, Xátiva, Spain. ${ }^{11}$ Hospital Virgen de la Salud, Toledo, Spain. ${ }^{12}$ Hospital Gregorio Marañón, Madrid, Spain. ${ }^{13}$ Hospital Puerta del Mar, Cadiz, Spain. ${ }^{14}$ Hospital 
Universitario de Vigo, Vigo, Spain. ${ }^{15}$ Hospital Marqués de Valdecilla, Santander, Spain. ${ }^{16}$ Hospital A Coruña Teresa Herrera, A Coruña, Spain. ${ }^{17}$ Hospital de Pontevedra, Pontevedra, Spain. ${ }^{18}$ Hospital Morales Meseguer, Murcia, Spain. ${ }^{19} \mathrm{Hospital}$ de Elche, Elche, Spain. ${ }^{20}$ Hospital Ramón y Cajal, Madrid, Spain. ${ }^{21}$ Hospital Provincial Castellón, Castellón de la Plana, Spain. ${ }^{22}$ Complejo Hospitalario de Jaén, Jaén, Spain. ${ }^{23}$ Hospital del Ferrol, Ferrol, Spain. ${ }^{24} \mathrm{Hospital}$ Regional Universitario, Málaga, Spain. ${ }^{25} \mathrm{Hospital}$ Miguel Servet, Zaragoza, Spain.

Received: 20 September 2018 Accepted: 16 November 2018

\section{Published online: 29 November 2018}

\section{References}

1. Siegel RL, Miller KD, Jemal A. Cancer statistics, 2015. CA Cancer J Clin. 2015;65(1):5-29.

2. De Angelis R, Sant M, Coleman MP, Francisci S, Baili P, Pierannunzio D, et al. Cancer survival in Europe 1999-2007 by country and age: results of EUROCARE-5-a population-based study. Lancet Oncol. 2014;15(1):23-34.

3. Bouvier A-M, Bossard N, Colonna M, Garcia-Velasco A, Carulla M, Manfredi S, et al. Trends in net survival from pancreatic cancer in six European Latin countries: results from the SUDCAN population-based study. Eur J Cancer Prev. 2017:S63-9. https://doi.org/10.1097/CEJ.00000000000000303.

4. Malvezzi M, Carioli G, Bertuccio P, Boffetta P, Levi F, La Vecchia C, et al. European cancer mortality predictions for the year 2018 with focus on colorectal cancer. Ann Oncol Off J Eur Soc Med Oncol. 2018;29(4):1016-22.

5. De Felice F, Musio D, Raffetto N, Tombolini V. Neoadjuvant strategy as initial treatment in resectable pancreatic cancer: concrete evidence of benefit. Anticancer Res. 2014;34(9):4673-6.

6. Conroy T, Bachet J-BB, Ayav A, Huguet F, Lambert A, Caramella C, et al. Current standards and new innovative approaches for treatment of pancreatic cancer. Eur J Cancer. 2016;57:10-22.

7. Ellenrieder V, König A, Seufferlein T, Konig A, Seufferlein T. Current standard and future perspectives in first- and second-line treatment of metastatic pancreatic adenocarcinoma. Digestion. 2016;94(1):44-9.

8. Conroy T, Desseigne F, Ychou M, Bouché O, Guimbaud R, Bécouarn Y, et al. FOLFIRINOX versus gemcitabine for metastatic pancreatic cancer. N Engl J Med. 2011;364(19):1817-25.

9. Von Hoff DD, Ervin T, Arena FP, Chiorean EG, Infante J, Moore M, et al. Increased survival in pancreatic cancer with nab-paclitaxel plus gemcitabine. N Engl J Med. 2013;369(18):1691-703.

10. Sohal DPS, Mangu PB, Khorana AA, Shah MA, Philip PA, O'Reilly EM, et al. Metastatic pancreatic cancer: american society of clinical oncology clinical practice guideline. J Clin Oncol. 2016;34(23):2784-96.

11. Ducreux M, Cuhna AS, Caramella C, Hollebecque A, Burtin P, Goere D, et al. Cancer of the pancreas: ESMO clinical practice guidelines for diagnosis, treatment and follow-up. Ann Oncol. 2015;26(Suppl 5):v56-68.

12. Le N, Vinci A, Schober M, Krug S, Javed MA, Kohlmann T, et al. Real-world clinical practice of intensified chemotherapies for metastatic pancreatic cancer: results from a pan-european questionnaire study. Digestion. 2017; 94(4):222-9.

13. Peixoto RD, Ho M, Renouf DJ, Lim HJ, Gill S, Ruan JY, et al. Eligibility of metastatic pancreatic cancer patients for first-line palliative intent nab-paclitaxel plus gemcitabine versus FOLFIRINOX. Am J Clin Oncol Cancer Clin Trials. 2017; 40(5):507-11.

14. Giordano G, Vaccaro V, Lucchini E, Musettini G, Bertocchi P, Bergamo F, et al. Nab-paclitaxel (Nab-P) and gemcitabine (G) as first-line chemotherapy (CT) in advanced pancreatic cancer (APDAC) elderly patients (pts): a "real-life" study. J Clin Oncol. 2015;33(3_suppl):424.

15. Ghosn M, Ibrahim T, Assi T, El Rassy E, Kourie HR, Kattan J. Dilemma of first line regimens in metastatic pancreatic adenocarcinoma. World J Gastroenterol. 2016;22(46):10124-30.

16. Therasse P, Arbuck SG, Eisenhauer EA, Wanders J, Kaplan RS, Rubinstein L, et al. New guidelines to evaluate the response to treatment in solid tumors. J Natl Cancer Inst. 2000;92(3):205-16.

17. National Cancer Institute. CTCAE v4.0 Archive. Common Terminology Criteria for Adverse Events (CTCAE). 2009. Available from: https:/evs.nci.nih. gov/ftp1/CTCAE/About.html. Cited 24 Jan 2018.

18. Goldstein D, El-Maraghi RH, Hammel P, Heinemann V, Kunzmann V, Sastre J, et al. Nab-paclitaxel plus gemcitabine for metastatic pancreatic cancer: long-term survival from a phase III trial. J Natl Cancer Inst. 2015;107(2):dju413.
19. Lo Re G, Santeufemia DA, Foltran L, Bidoli E, Basso SMM, Lumachi F. Prognostic factors of survival in patients treated with nab- paclitaxel plus gemcitabine regimen for advanced or metastatic pancreatic cancer: a single institutional experience. Oncotarget. 2015;6(10):8255-60.

20. De Vita F, Ventriglia J, Febbraro A, Laterza MM, Fabozzi A, Savastano B, et al. NAB-paclitaxel and gemcitabine in metastatic pancreatic ductal adenocarcinoma (PDAC): from clinical trials to clinical practice. BMC Cancer. 2016;16(1):1-8.

21. Giordano G, Vaccaro V, Lucchini E, Bertocchi P, Bergamo F, Musettini G, et al. Analysis of prognostic factors in advanced pancreatic cancer (APDAC) patients (pts) undergoing to first-line nab-paclitaxel (Nab-P) and gemcitabine (G) treatment. J Clin Oncol. 2015;33(3_suppl):412.

22. Tas F, Sen F, Keskin S, Kilic L, Yildiz I. Prognostic factors in metastatic pancreatic cancer: older patients are associated with reduced overall survival. Mol Clin Oncol. 2013;1(4):788-92.

23. Montes A, Villarroel P, Ayerbes M, la Gómez JC, Aldana G, Tuñas L, et al. Prognostic and predictive markers of response to treatment in patients with locally advanced unresectable and metastatic pancreatic adenocarcinoma treated with gemcitabine/nab-paclitaxel: results of a retrospective analysis. J Cancer Res Ther. 2017;13(2):240

24. Huang L, Jansen L, Balavarca Y, Molina-Montes E, Babaei M, van der Geest L, et al. Resection of pancreatic cancer in Europe and USA: an international large-scale study highlighting large variations. Gut. 2017. https://doi.org/10. 1136/gutjnl-2017-314828.

25. Hamada T, Nakai Y, Yasunaga H, Isayama H, Matsui H, Takahara N, et al. Prognostic nomogram for nonresectable pancreatic cancer treated with gemcitabine-based chemotherapy. Br J Cancer. 2014;110(8):1943-9.

26. Goldstein D, Von Hoff DD, Chiorean EG, Reni M, Tabernero J, Ramanathan RK, et al. Nomogram for predicting overall survival (OS) in patients (pts) treated with nab-paclitaxel (nab-P) plus gemcitabine (Gem) or Gem alone for metastatic pancreatic cancer (MPC). J Clin Oncol. 2017;35(15_suppl):4109. http://ascopubs.org/doi/abs/10.1200/JCO.2017.35.15_suppl.4109.

\section{Ready to submit your research? Choose BMC and benefit from:}

- fast, convenient online submission

- thorough peer review by experienced researchers in your field

- rapid publication on acceptance

- support for research data, including large and complex data types

- gold Open Access which fosters wider collaboration and increased citations

- maximum visibility for your research: over $100 \mathrm{M}$ website views per year

At BMC, research is always in progress.

Learn more biomedcentral.com/submissions 